\title{
Emergency medical services witnessed pulseless electrical activity [PEA] has only marginal survival benefit compared to layperson witnessed PEA
}

\section{Ari Salo M.D.; Tuukka Puolakka M.D. PhD; Markku Kuisma M.D. PhD}

Section of Emergency Medical Services, Department of Emergency Medicine, Helsinki University Hospital and University of Helsinki, Finland

\section{Background}

Time delay to CPR and first defibrillation has been shown to decrease survival from cardiac arrest caused by ventricular fibrillation. Survival from layperson witnessed PEA however has not been shown to have a similar time dependent correlation. We wanted to investigate whether emergency medical services (EMS) witnessed PEA has a survival benefit compared to layperson witnessed PEA in out-of-hospital cardiac arrest (OHCA).

\section{Materials and methods}

We performed a retrospective observational study from the OHCA registry of Helsinki, Finland. All layperson and EMS witnessed cardiac arrests with PEA as the initial rhythm were included from 1.1.1997 to 31.12.2017. Survival from the hospital was the primary end point for the study.

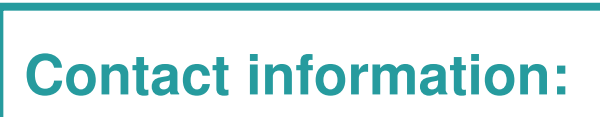

\section{Results}

During the study period, 7475 OHCAs occurred. Resuscitation was attempted in 5028 cases $(67.3 \%)$. There were 1054 layperson witnessed and 523 EMS witnessed resuscitation attempts with PEA as the initial rhythm (Table 1). 61 EMS witnessed PEA patients $(11.7 \%)$ survived from the hospital compared to 91 (8.6 \%) layperson witnessed PEA patients $(\mathrm{p}=0.057)$ (Figure1).

Survival with good neurological outcome (cerebral performance category score of 1 or 2) was only moderately higher in EMS witnessed PEA $(9.9 \%)$ compared to layperson witnessed PEA (6.3 \%) with statistical significance $(p=0.011)$. There was no difference in one-year survival (EMS witnessed $9.8 \%$, layperson witnessed $7.1 \%$, $\mathrm{p}=0.076$ ).

Prehospital emergency physician presence at the time of EMS witnessed PEA ( $n=161)$ did not improve survival from the hospital (11.2\%) compared to delayed arrival or other units (BLS and/or ALS) being present $(11.9 \%)$ at the time of arrest $(\mathrm{p}=0.627)$.

\section{Conclusions}

Immediate resuscitation by EMS personnel in EMS witnessed PEA seems to offer only modest survival benefit compared to layperson witnessed PEA in OHCA.

\begin{tabular}{|l|c|c|c|}
\hline \multicolumn{4}{|l|}{ Table 1. Comparison of EMS and layperson witnessed PEA resuscitation attempts } \\
\hline Variable & EMS witnessed $(\mathbf{n = 5 2 3 )}$ & Layperson witnessed (n=1054) & p-value \\
\hline Age, years & $65.0( \pm 16.0)$ & $67.3( \pm 16.8)$ & 0.01 \\
\hline Male & $63.9 \%$ & $62.0 \%$ & 0.507 \\
\hline ROSC achieved & $46.6 \%$ & $43.6 \%$ & 0.282 \\
\hline ROSC, min & $10(5-16)$ & $20(16-25)$ & $<0.0001$ \\
\hline Epinephrine used & $83.6 \%$ & $80.2 \%$ & 0.114 \\
\hline Epinephrine, mg & $2.0(1-5)$ & $2.0(1-4)$ & 0.0001 \\
\hline Survival to hospital & $35.2 \%$ & $34.5 \%$ & 0.822 \\
\hline Survival from hospital & $11.7 \%$ & $8.6 \%$ & 0.057 \\
\hline Patient age is given as mean $( \pm$ standard deviation) and other variables using median (interquartile range). \\
\hline
\end{tabular}

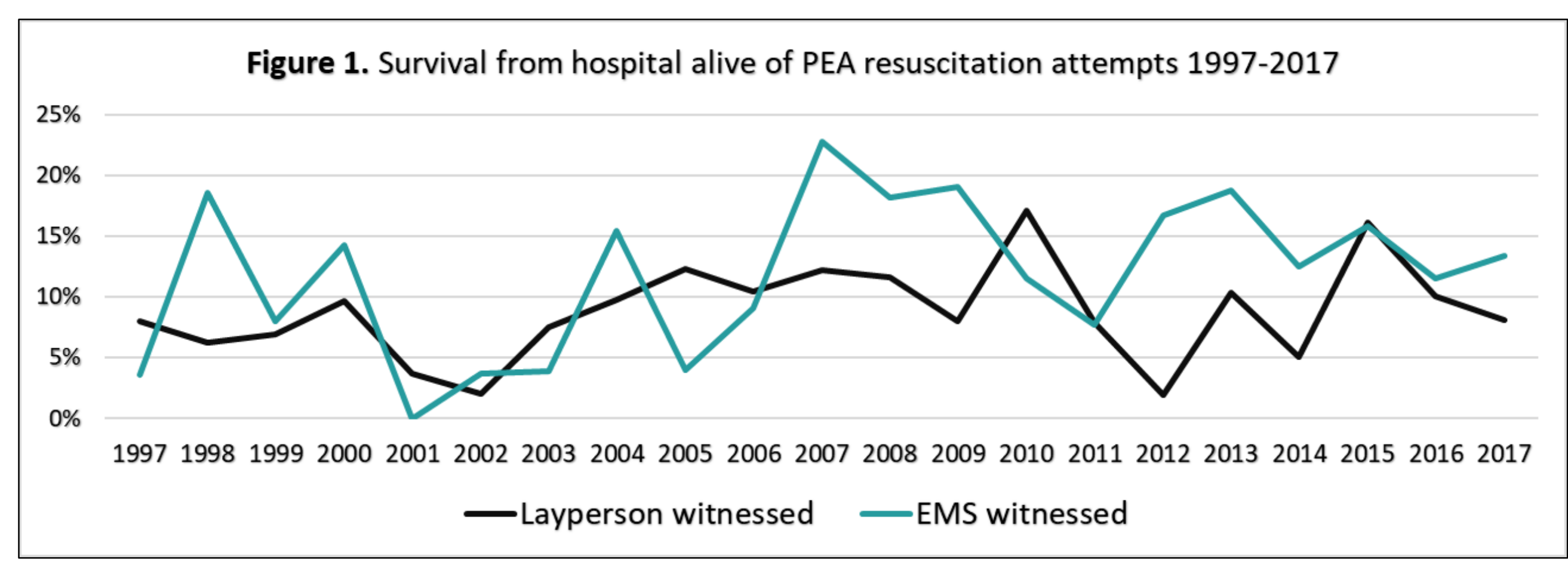

